\title{
ANALOG FRONT-END ELECTRONICS FOR BEAM POSITION MEASUREMENT ON THE BEAM HALO MEASUREMENT ${ }^{*}$
}

\author{
R. B. Shurter, T. Cote, J. D. Gilpatrick, Los Alamos National Laboratory, Los Alamos, NM 87545, \\ USA
}

\section{Abstract}

Enhancements have been made to the log-ratio analog front-end electronics based on the Analog Devices 8307 logarithmic amplifier as used on the LEDA accelerator. The dynamic range of greater than $85 \mathrm{~dB}$, has been extended to nearly the full capability of the AD8307 from the previous design of approximately $65 \mathrm{~dB}$ through the addition of a $350 \mathrm{MHz}$ band-pass filter, careful use of ground and power plane placement, signal routing, and power supply bypassing. Additionally, selection of highisolation RF switches (55dB) has been an integral part of a new calibration technique, which is fully described in another paper submitted to this conference [1]. Provision has also been made for insertion of a first-stage low-noise amplifier for using the circuit under low-signal conditions. configured to conduct a beam halo experiment.

Beam diagnostics include fifteen beam position monitors in the 52-quadrupole magnet focusingdefocusing (FODO) lattice, along with wire scanners, beam-loss monitors and current monitors [7]. The nature of the experiment places a requirement for additional dynamic range on the BPM electronics. Beam debunching, which occurs as the beam is transported through the lattice, reduces the signal power by about 40 $\mathrm{dB}$ from the last BPM with respect to the first.

\section{CIRCUIT DESCRIPTION}

Referring to the block diagram in Figure 1, signals from the four electrodes of the beam position monitors are band-pass filtered and input to the AD8307 log amplifier

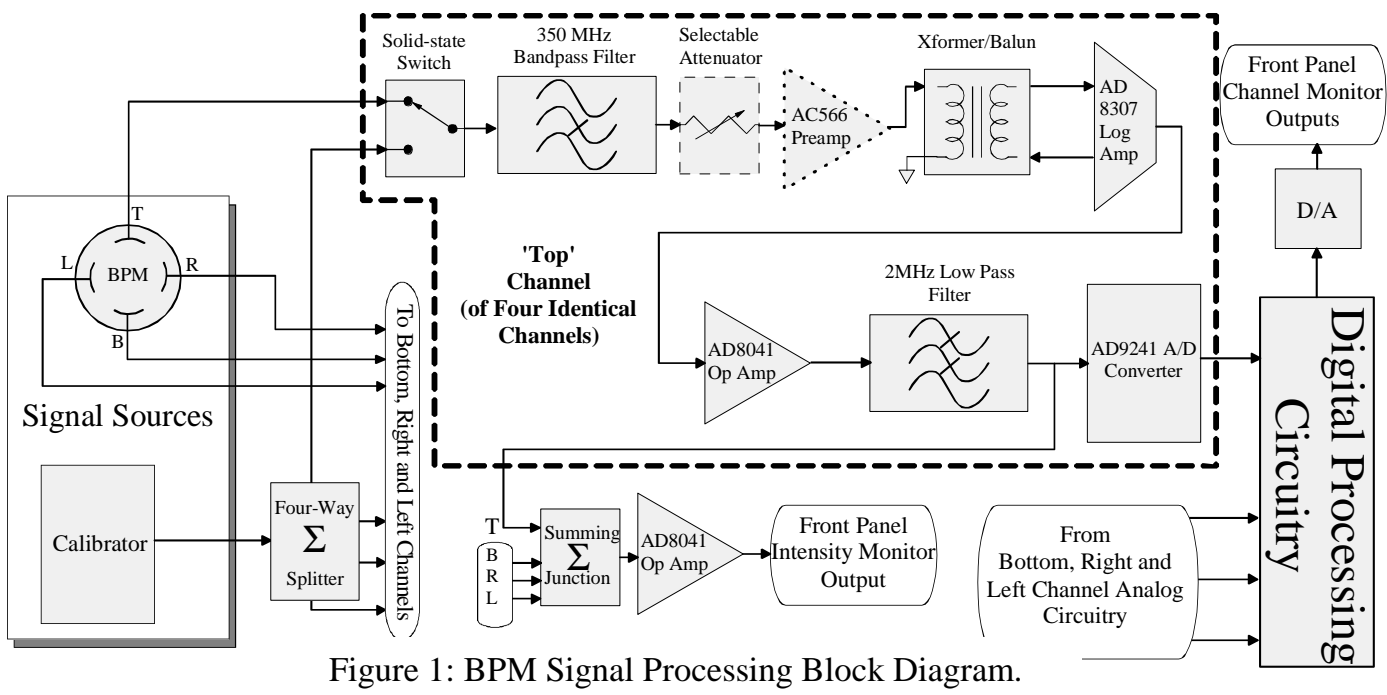

\section{BACKGROUND}

A BPM signal processor based on the AD8307 was previously used by us on the Low Energy Demonstration Accelerator (LEDA), part of the Accelerator Production of Tritium (APT) project at Los Alamos. Experience with that system was reported at the 2000 Beam Instrumentation Workshop [2]. Several of the problems encountered with that system have been corrected, as well as new capabilities added, with the resultant system being presented here. Currently the LEDA accelerator is

* Supported by US DOE, Office of Defense Programs, and the Office of Nuclear Energy, Science and Technology. through a transformer balun, which is required because of the differential inputs with an impedance of $1 \mathrm{k} \Omega$ shunted by $1.4 \mathrm{pf}$ to common. The circuit layout can accommodate a Minicircuits Lab fixed attenuator (PAT-X) and/or a Cougar Components AC566 pre-amplifier [8] installed between the filter and the transformer. This attenuatoramplifier combination allows us to adjust the signal highend for the log amp's maximum input of approximately $+17 \mathrm{dBm}$, thereby obtaining the greatest signal to noise ratio and dynamic range (approximately $92 \mathrm{~dB}$ is specified for the AD8307.)

The log amp output is then amplified, scaled and offsetadjusted by an AD8041 amplifier stage for a 0 to approximately $4.6 \mathrm{~V}$ range. The signal is then low-pass 
filtered to a $200-\mathrm{KHz}$ bandwidth and digitized by an AD9241 14-bit analog to digital converter. Only 12 bits of the A/D is used, giving a resolution of $5 \mathrm{~V} / 4096=1.2$ $\mathrm{mV} /$ count. The result of one channel's raw (calibrator errors not corrected) calibration response, as seen on a LabVIEW PC display, is given in figure 2.

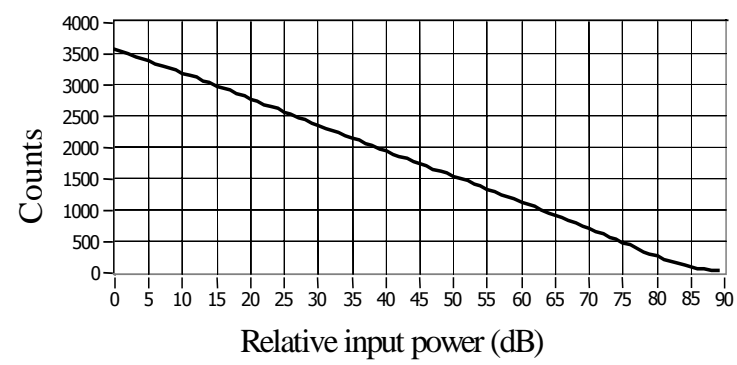

Figure 2. Calibration display

\section{IMPROVMENTS}

The improvements to the previous design include:

- Correction of overlapping digital and analog power and ground planes. Signal line re-routing to eliminate crossing gaps in the planes and/or keeping the planesignal types coplanar.

- Addition of an RF shield around the RF components.

- Input filter change from low-pass to band-pass. This resulted in the largest dynamic range gain.

- Impedance matching all input RF traces and careful attention to shortness of trace length between components and their bypass capacitors.

- Elimination of one baseband gain stage.

\subsection{Input Filter}

Our previous design used a low-pass filter at the RF input. We found that most of the debilitating noise we were experiencing could be eliminated by using a bandpass filter custom made by TTE, Inc [3].

\subsection{Circuit Layout}

Attention was given to the routes return currents traveled in order to make sure that those currents did not induce signals in low-signal parts of the circuitry. Because we were not absolutely certain about this, we provided jumper pads for tying the analog and digital ground planes together at various points. Additionally, the recommended printed circuit layout for the Analog Devices AD9241 A/D converter was followed. The AD9241 product specifications define how the analog and digital ground planes are separated under and around the part [4][5].

Large co-planar power and ground planes were used to provide low-impedance power and return paths, as well as to increase the capacitive coupling between them for shunting unwanted signals. Care was taken to make sure signal lines were not routed between these planes.
Signal traces were never allowed to cross gaps in ground planes, as this would create current loops, thereby inducing currents in nearby components [6].

\subsection{Dynamic Range improvement}

Figure 3. shows a graph of the dynamic range of the AFE circuit as previously used on the LEDA accelerator. Figure 4. shows the improved range of the circuit used on the HALO measurement.

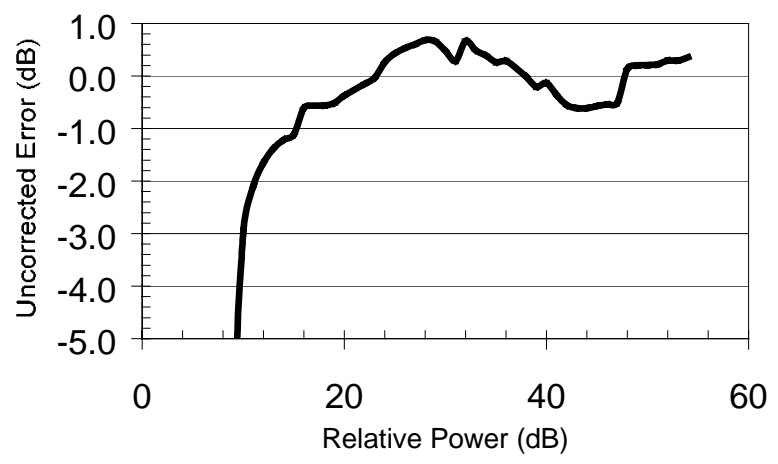

Figure 3. LEDA BPM AFE Linearity

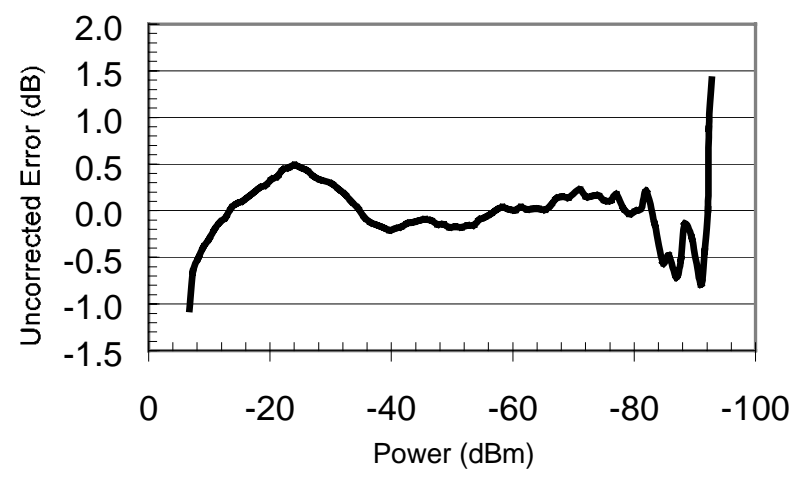

Figure 4. HALO BPM AFE Linearity

\section{CONCLUSION}

The Analog Devices AD8307 Logarithmic amplifier's superior $92 \mathrm{~dB}$ dynamic range and $500 \mathrm{MHz}$ bandwidth makes an excellent beam signal detector as compared to other techniques, e.g.. synchronous detection, as long as careful attention is given to following good noise reduction techniques.

Since the logarithmic non-conformance of this type of signal processing can introduce deterministic errors of up to $+/-0.5 \mathrm{~dB}$, calibration is used to further enhance accuracy [9].

Our experience with the BPM VXI processing electronics modules has shown them to be highly reliable. The few failures we have experienced have been due to mis-soldered components, mother-board problems, and in two cases, defective VXI crates. 


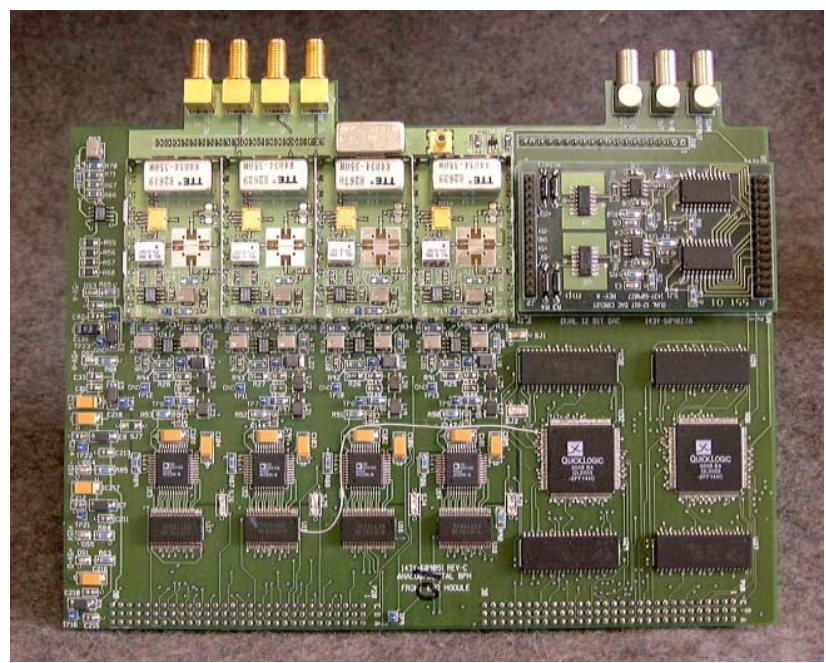

Figure 5. HALO BPM AFE Circuit Board

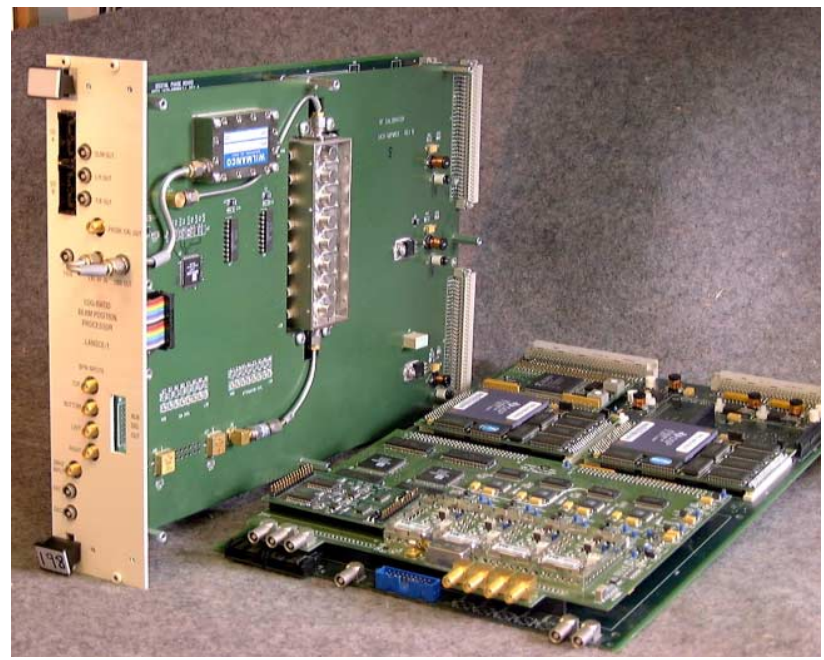

Figure 6. HALO BPM VXI Module with AFE on Motherboard

\section{ACKNOWLEDGMENT}

The authors wish to thank John Power for his initial work on this measurement design, as well as his continued help.

\section{REFERENCES}

[1] D. Barr, J. D. Gilpatrick, R. Shurter, "Upgrade to Initial BPM Electronics Module and Beamline Components for Calibration of the LEDA Beam Position Measurements," published at this conference.

[2] R.B. Shurter, J. D. Gilpatrick, J. Power, "BPM Analog Front-End Electronics Based on the AD8307 Log Amplifier," $9^{\text {th }}$ Beam Instrumentation Workshop, Cambridge, MA, May 8-11, 2000.

[3] TTE, Inc., http://www.tte.com/. Filter specificationsModel number: K4034-350M-20M-50-484, 3-pole chebyshev, $\mathrm{F}_{0}: 350 \mathrm{MHz}$, Insertion loss @ $\mathrm{F}_{0}: 5 \mathrm{~dB}$ max., Rejection (typical): $-40 \mathrm{~dB} @ 270 \mathrm{MHz}$ and 360MHz, Impedance: $50 \mathrm{ohms}$.

[4] Analog Devices, Inc., www.analogdevices.com AD9241 specification

[5] Analog Devices, Inc., AN-404 Application note.

[6] T. Van Doren, "Circuit Board Layout to Reduce Electro-magnetic Emission and Susceptibility, National Technological University live video course \#MC00032802, March 28, 2000.

[7] J. D. Gilpatrick, et al., "Experience with the Low Energy Demonstration Accelerator (LEDA) Halo Experiment Beam Instrumentation," published at this conference.

[8] Cougar Components, Inc., http://www.cougarcorp.com/

[9] D. Barr, J. D. Gilpatrick, R. Shurter, "Upgrade to Initial BPM Electronics Module and Beamline Components for Calibration of the LEDA Beam Position Measurements," published at this conference. 